\title{
A neural model for chronic pain and pain relief by extracorporeal shock wave treatment
}

\author{
Othmar J. Wess
}

Received: 22 April 2008 / Accepted: 19 September 2008 / Published online: 7 October 2008

(C) The Author(s) 2008. This article is published with open access at Springerlink.com

\begin{abstract}
The paper develops a new theory of chronic pain and pain relief by extracorporeal shock wave treatment. Chronic pain without underlying anatomical disorder is looked at as a pathological control function of memory. Conditioned reflexes are considered to be engraved memory traces linking sensory input of afferent signals with motor response of efferent signals. This feature can be described by associative memory functions of the nervous system. Some conditioned reflexes may cause inappropriate or pathological reactions. Consequently, a circulus vitiosus of pain sensation and muscle and/or vessel contraction is generated when pain becomes chronic (pain spiral). The key feature is a dedicated engram responsible for a pathological (painful) reaction. The pain memory may be explained by the concept of a holographic memory model published by several authors. According to this model it is shown how nervous systems may generate and recall memory contents. The paper shows how extracorporeal shock wave treatment may reorganize pathologic memory traces, thus giving cause to real and permanent pain relief. In a generalized manner, the idea of associative memory functions may help in the understanding of conditioning as a learning process and explain extracorporeal shock wave application as an efficient treatment concept for chronic pain. This concept may open the door for new treatment approaches to chronic pain and several other disorders of the nervous system.
\end{abstract}

O. J. Wess ( $\square)$

Storz Medical AG, Lohstampfestrasse 8,

8274 Tägerwilen, Switzerland

e-mail: wess.othmar@storzmedical.com
Keywords ESWT - Chronic pain · Pain memory · Shock wave treatment $\cdot$ Associative memory . Holographic brain model · CPPS

\section{Introduction}

Chronic pain distresses are among the most frequent diseases in the western world. Patients often suffer from severe pain sensations such as frozen or calcified shoulder, tennis elbow, lower back pain, heel spurs etc. Many patients remain without any improvement in spite of injections of corticoids, massages and other treatment modalities. In urology the chronic pelvic pain syndrome (CPPS) and remaining pain symptoms after successful stone clearance may stand for chronic pain without clear anatomical disorders.

Since 1980 shock waves have been frequently used for fragmentation of body concrements. Extracorporeal shock wave lithotripsy (SWL) is the method of choice for fragmentation of urinary stones and is also utilized for disintegration of several other types of body calculi. In the 1990s, the idea of fragmentation of calcified deposits led to shock wave applications for indications such as tendinosis calcarea, tennis elbow and others. During recent years, extracorporeal shock wave treatment (ESWT) of soft tissue pain close to bone has gained significant interest in Europe and the USA.

Surprisingly, most extracorporeal shock wave treatments in orthopedics resulted in significant reduction of pain even if the calcium deposit, primarily, could not be disintegrated. It turned out that shock wave treatment shows a stimulating and healing effect beyond fragmentation of body calculi.

Typically, shock wave treatment of chronic pain is performed by application of hundreds to a few thousands of 
shock wave pulses with a repetition frequency of 1-4 pulses per second to the area of highest pain sensation. The shock wave energy is relatively low, approximately $1 / 10$ of the energy levels used for stone fragmentation. Shock waves are applied extracorporeally, by coupling the therapy head of the device to the painful area. They may be focused or not to reach the appropriate area of interest within the body or at the surface. Shock waves may be guided by aid of ultrasonic or fluoroscopic localization devices. Most often they are guided by the feedback of the patient himself by responding to the level of pain sensation during the treatment (biofeedback).

The application of shock waves is slightly painful and causes an acceptable but usually not negligible discomfort. Local anesthesia has been used to reduce pain sensation during ESWT; however, it turned out that it reduced the healing effect of shock waves simultaneously [1]. Usually the treatment is split into several sessions (1-6) with a time interval of a few days or weeks.

This treatment regime or a similar one is used for chronic pelvic pain as well as shoulder pain, heel spur and angina pectoris for example.

As a mechanism of pain relief by extracorporeal shock wave treatment several theories are discussed $[2,3]$.

1. Shock waves stimulate the nociceptors to fire high-frequent nerve impulses (hyperstimulation). Propagation of nerve impulses is blocked according to the gate-control theory [4].

2. Shock waves distort parts of or the total cell membrane. The nociceptors cannot build up a generator potential; thus pain sensation is avoided.

3. Shock waves change the chemical environment of the cell membranes by generating free radicals, which in turn result in pain-inhibiting chemicals in the vicinity of the cells.

Recent publications [5, 6] claim revascularization stimulated by shock waves to be the basic mechanism of pain relief and tissue healing.

Pain relief is not the only effect associated with ESWT. Lohse-Busch et al. [7] reported reduction of muscular rigidity and spastic co-contractures after shock wave application in patients suffering from tetraspastic disorders due to infantile cerebral palsy. Manganotti et al. [8] demonstrated a long-term effect of shock wave therapy on patients affected by stroke. Russo et al. [9] saw a stimulation of micro-vascularization after shock wave treatment. CPPS [10], angina pectoris and wound healing [11] are promising new fields of shock wave application. The underlying mechanism, however, is still speculative.

This paper describes a potential neural mechanism for reorganization of pathological reflex patterns by extracorporeal shock wave therapy for chronic pain relief.

\section{Associative memory model for establishing reflex functions}

Conditioned reflexes are considered engraved memory control functions linking sensory input of afferent nervous system with motor output of efferent signals. Associative memory is a very efficient and fast mechanism enabling living beings to react fast and appropriately to changing environmental conditions. An overwhelming majority of our experiences stored within the memory is well matched and optimally responds to daily living conditions. Some conditioned reflexes, however, may cause inappropriate or pathological reactions. Chronic pain, for example, without underlying anatomical disorders is considered a pathological control function. An interaction between afferent sensor input and efferent motor output is postulated to form a reflex-like response. Accordingly, a circulus vitiosus of pain sensation and muscle and/or vessel contraction is generated when pain becomes chronic.

Learning model of reflex functions on the concept of engrams

The term associative pain memory reflects the fact that pain generates its own associative memory effect, linking painspecific sensory inputs to particular motor outputs as sketched in Fig. 1.

If we consider, for example, pain sensation in the shoulder, consequently, shoulder muscles contract for pain relief by aid of protective posture. The modified muscle tonus causes reduction of blood circulation and metabolism in that particular area, which in turn may induce additional pain. If this reflex bow lasts significantly long or if it is very intense, pain sensation and contracted muscles are linked and engraved as an associative pair of items. Whenever a pain sensation is present, the muscle tonus is affected and vice versa. Thus, a feedback loop with positive reverbera-

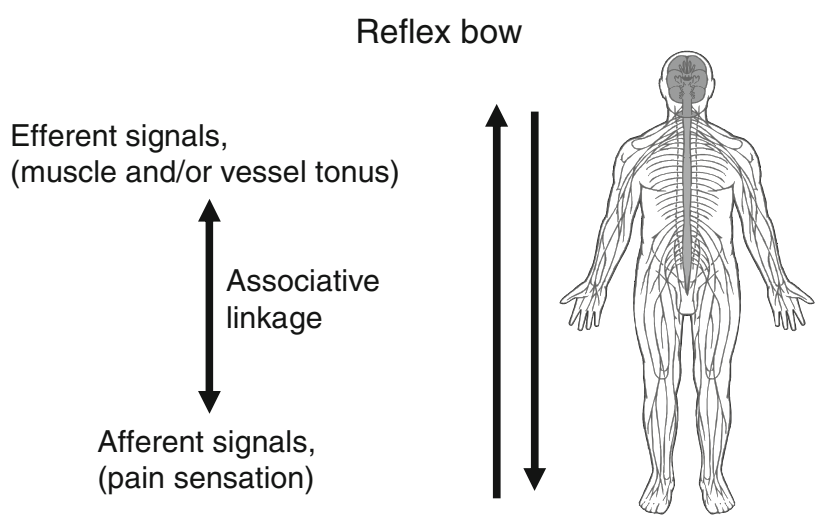

Fig. 1 Basic concept of associative memory linking pain sensation and muscle and/or vessel tonus associatively 
tion is established. The linking memory effect is located somewhere on the nervous pathway in between sensor input and motor output depending on the involved nervous structure Fig. 2.

As known from learning experiments in general, it is assumed that memory effects are based on modifications of the synaptic junctions between nervous cell ensembles within the CNS. Storing information in the long-term memory, usually, requires strong and repeated stimulation of the involved neurons on different hierarchic levels of the CNS. The result of such a successful learning procedure is considered an engram.

This type of associative memory is a basic factor for the enormous efficiency of the human brain.

The type of chronic pain we are interested in is considered to be stored as an engram in the above-mentioned sense. Pain signals modify an ensemble of nerve cells of the peripheral nervous system (PNS) as well as in the central nervous system (CNS), forming a pain memory for this specific pain sensation which, in turn, links a dedicated motor response to the effecting organs such as muscles and vessels (Fig. 3).

The hypothesis of this paper can be described as follows:

When acute pain develops into chronic pain, sensor input and motor output are stored associatively in the PNS and/or CNS and act in a feedback type circle.

From that particular point of time on, the cause of that (chronic) pain has shifted from the organ itself to higher levels of the PNS or CNS. Consequently, successful treatment regimes must affect the pathological reflex bow and erase the particular memory instead of modifying the organ itself.

Information storage by modification of synapses

Nerve signals are transmitted by means of action potentials along axons and chemical substances within the synaptic junctions. If the signals are strong enough or if many adja-

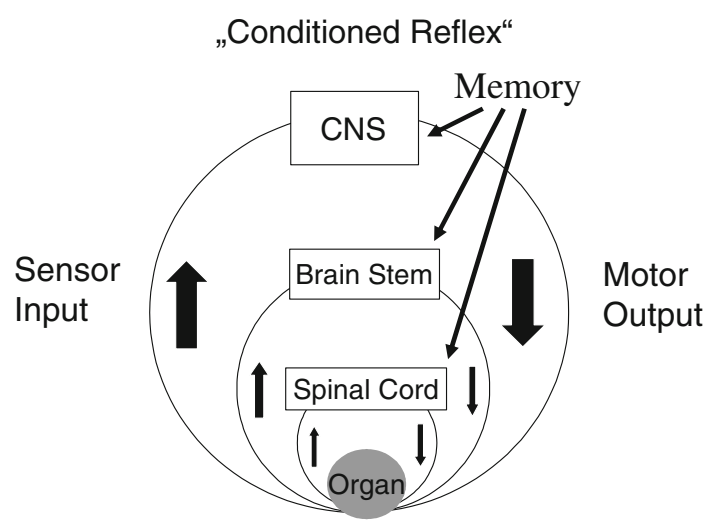

Fig. 2 Feedback circuit of sensor input and motor output

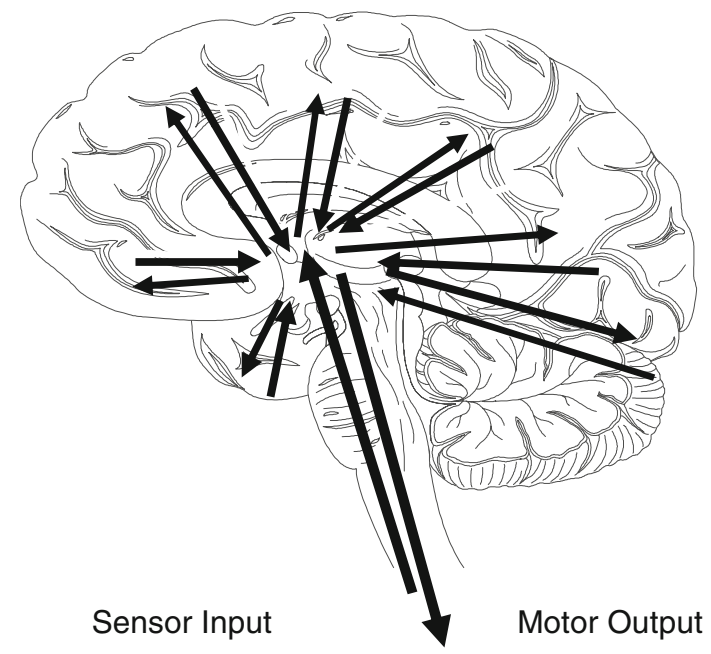

Fig. 3 Schematic view of input and output pathways from peripheral organs to the CNS

cent synapses are releasing transmitter substances from their vesicles simultaneously, the contacted nerve cell in turn will fire subsequent electrical signals. They propagate via the axons to succeeding nerve cells. This may result in the appropriate reaction of the muscles in order to get rid of the primary pain. For example, a person may touch the flame of a burning candle and retract the finger to get out of this painful situation. This is considered as a "normal" reaction. In some cases, however, the painful situation may continue for quite a while so that the involved synaptic junctions may be activated in a repeat manner. It is well known that frequently activated synapses transmit signals with a lower threshold so that after the "training or learning process" even very small inputs may activate the following cells. The synaptic strength of particular synapses is increased (long-term potentiation, LTP). Thus, a reflex bow of connecting sensor input (pain) with a specific motor response (muscle or vessel contraction) is established. Trained sequences of (afferent) sensation and (efferent) motor response are the basis of all trained skills. It becomes a problem only if it develops into a reverberating selfenhancing feedback loop as in chronic pain.

Associative memory and its impact on pain therapy

In cases of chronic pain without organic reasons the theory of associative memory draws the attention away from the painful organ to a higher hierarchic memory level. The organ itself is no longer the region of primary therapeutic interest but the according pain memory located in the modified synaptic structure within the related reflex bow. Direct mechanistic view is replaced by looking at higher control functions of the nervous system and its memory potential. Obviously, this idea leads to a radical change of therapeutic approaches of chronic pain distresses. Therapeutic treat- 
ment modalities are no more focused on specific organs under pain but on pain memory. In other words: Pain treatment strategies are directed towards reorganization of the memory structure instead of direct (physical or chemical) modification of the organ.

The existence of memory capabilities in general is obvious in humans and highly developed living beings; however, the knowledge about the underlying mechanism is fragmentary and requires further investigation.

Several brain models were developed to shine some light on the subject and to enhance understanding of memory functions.

\section{Holographic memory model}

Neuronal memory function of the CNS can be described by a variety of different brain models. Some brain functions can be simulated by neural nets taking into account some specific features of the synaptic junctions such as adaptation, non-linear behavior and response thresholds [12]. There are non-holographic models [13] as well as holographic models. They make use of correlation functions which are assumed to be performed by neural systems [14].

A holographic model, in particular, matches associative memory functions of the brain quite well. There are striking analogies between a holographic system and the memory functions of information processing by the CNS.

Neural and holographic analogies are listed below:

\begin{tabular}{|c|c|}
\hline CNS/PNS & Hologram \\
\hline Parallel information processing & $\begin{array}{l}\text { Parallel processing of } \\
\text { two- or three-dimensional } \\
\text { information }\end{array}$ \\
\hline Divergence of afferent signals & $\begin{array}{l}\text { Divergent waves } \\
\quad \text { (virtual images) }\end{array}$ \\
\hline Convergence of efferent signals & $\begin{array}{l}\text { Convergent waves } \\
\text { (real images) }\end{array}$ \\
\hline $\begin{array}{l}\text { Memory contents not precisely } \\
\text { localized [15] }\end{array}$ & $\begin{array}{l}\text { Spatial distribution of stored } \\
\text { information }\end{array}$ \\
\hline $\begin{array}{l}\text { Same group of neurons stores } \\
\text { different information }\end{array}$ & $\begin{array}{l}\text { Separate storage of multiple } \\
\text { holograms on the same } \\
\text { storage medium }\end{array}$ \\
\hline $\begin{array}{l}\text { Pattern recognition independent } \\
\text { from translation, magnitude } \\
\text { and direction }\end{array}$ & $\begin{array}{l}\text { Parallel translation invariant } \\
\text { information processing }\end{array}$ \\
\hline $\begin{array}{l}\text { Content of long term memory } \\
10^{10}-10^{14} \mathrm{bit}\end{array}$ & Storage capacity $10^{11}-10^{13}$ bit \\
\hline $\begin{array}{l}\text { Recall of pairs of connected } \\
\text { events, associative learning }\end{array}$ & $\begin{array}{l}\text { Associative storage } \\
\text { and reconstruction }\end{array}$ \\
\hline $\begin{array}{l}\text { Sequential recall of linked } \\
\text { memory chains }\end{array}$ & $\begin{array}{l}\text { Configuration of associative } \\
\text { time chains }\end{array}$ \\
\hline Plasticity of memory & Dynamics of memory response \\
\hline
\end{tabular}

The idea of a holographic brain model was developed by several authors [16-20]. Associative memory chains based on non-linear concepts and three-dimensional storage mediums were developed by Mager et al. [21], Wess et al. [22].

\section{Neuro-holographic memory model}

Holographic brain models correlate well with associative memory functions of the brain. The crucial question is whether we can find biological structures capable of performing analog memory functions based on physiological features of the CNS. If so, we may look for appropriate therapeutic measures for chronic pain management.

Holographic storage and reconstruction of information is based on interference of coherent waves (as generated by lasers) to generate a time-invariant interference patterns, a so-called hologram. The neural counterpart of coherence is a synchrony of neural oscillatory actions as described by Gray et al. [23], Engel and Singer [24]. The underlying mechanism of both systems (holographic as well as neural) is the ability to perform correlation functions on spatial patterns.

The concept of holographic associative pain memory (Fig. 4) may lead the way to develop optimized therapeutic strategies.

Networked layers of nerve cells provide a basis for a systems-theoretical approach.

Excitation of sensory nerve cells leads to action potentials generated in higher layers of nerve cells. The excitation pattern is laterally widened (spread) by passing following layers of neurons up to the sensory cortex. Motor response, on the other hand, may be concentrated on a specific group of muscles, activated by converging signals

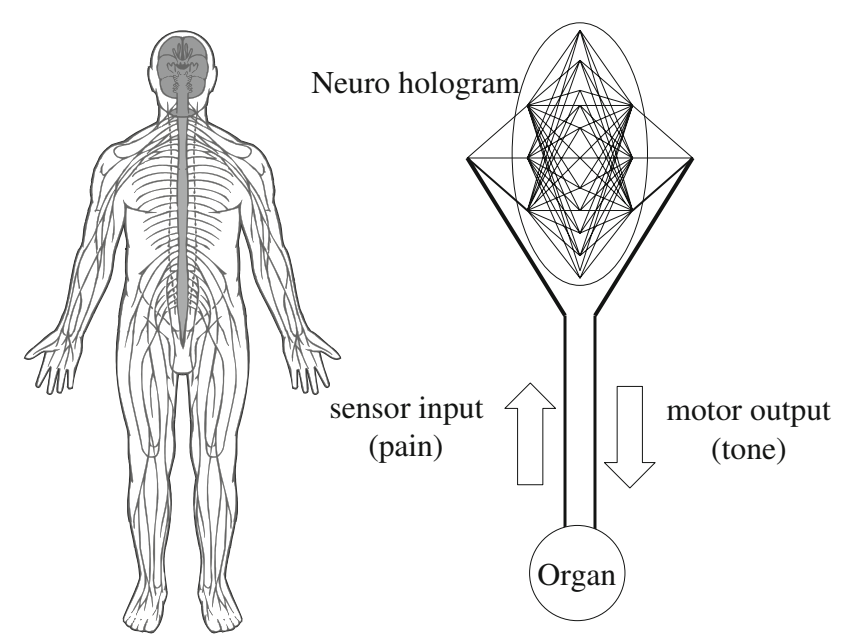

Fig. 4 Neuro hologram formation (schematic) 
controlled by an extended area of the motor cortex. The principle of divergence and convergence is known from physiology text books and is well mimicked by holographic storage and retrieval.

Analogous to the holographic model, interference of different input/output signals may take place on all involved layers of neurons as schematically depicted in Fig. 5.

Since neurons are activated only if a certain threshold of excitation is surpassed, only those neurons may respond by generating action potentials, which were activated by two or more simultaneously arriving signals. A spatial interference pattern is generated and transmitted through a sequence of neuronal layers localized anywhere in between sensor input and motor output. Figure 6 depicts this situation schematically. Only if the action potentials (gray and black dots) of two activated cells of the first layer simultaneously arrive at the cells of the subsequent layer, the thresholds of these cells are exceeded and the following cells (black) are activated. Single asynchronously arriving signals stay below the cell's thresholds and do not generate succeeding action potentials. By aid of the threshold behavior of neurons, spatial neural interference patterns may be established analogous to interference of coherent waves in holography.

Frequently used synapses are modified as mentioned previously and, thus, provide a mechanism to memorize (store) spatial interference patterns such as repeated associative sensor input/motor output patterns. In cases of chronic pain diseases pain sensation and muscle contraction may be permanently stored as a synaptic threshold modification pattern on various hierarchic levels of the CNS.

The concept of synaptic memory is based on the following findings and assumptions:

- Metabolism of intensely stimulated synapses is enhanced (long-term potentiation, LTP)

- Repeatedly stimulated synapses facilitate transmission of signals

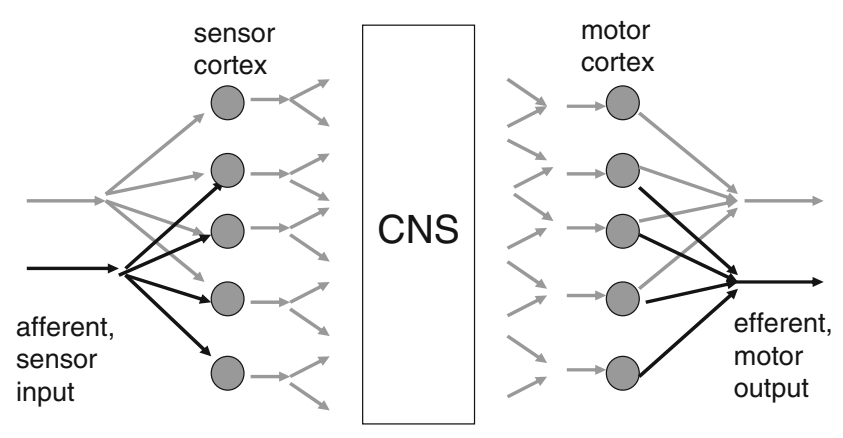

Fig. 5 Divergence and convergence of nerve signals by transmission through several layers of neurons

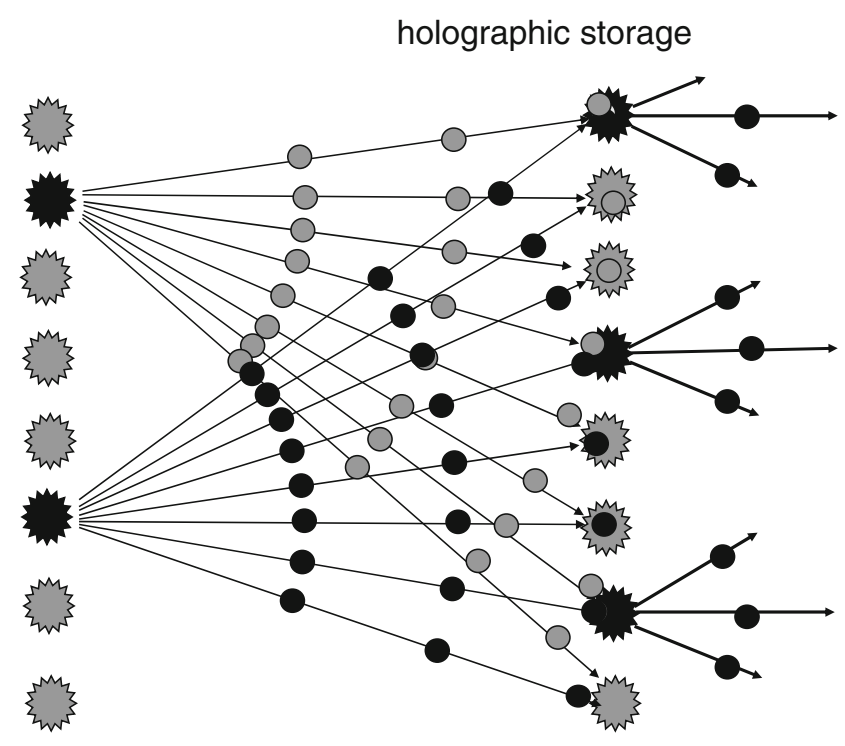

Fig. 6 Formation of neural interference pattern

- Neural interference patterns are permanently stored as excitability (threshold) pattern of large numbers of cells and synapses.

\section{Hypothetic mechanism of shock wave therapy}

Does the concept of associative holographic pain memory provide any idea how to cure chronic pain distresses in general and/or a consistent theory of ESWT? Which mechanism explains the selective erasure of an isolated pathological reflex pattern between pain sensation and muscle/vessel contraction? The problem seems to be too complex for a simple answer since the location of the pathology is considered not in the painful organ itself anymore but is diffusely spread over extended areas as well as over several levels of the PNS/CNS. How are isolated reflex patterns selectively erased without sacrificing the total memory content? The neuro-holographic model may provide a solution to the problem.

Healing by selective erasing of pathologic reflex patterns

Holography is based on storage of interference patterns and reconstruction by interaction of an input signal with the stored pattern. The strength of the associatively retrieved output pattern depends on the quality of the interference pattern, specifically on the depth of modulation, as shown in Fig. 7.

A characteristic feature of a hologram is, as listed above, that several interference patterns may be stored within the same storage medium by simply storing one pattern above the other. Reconstruction of different patterns is done by 
Waves of Equal Amplitudes

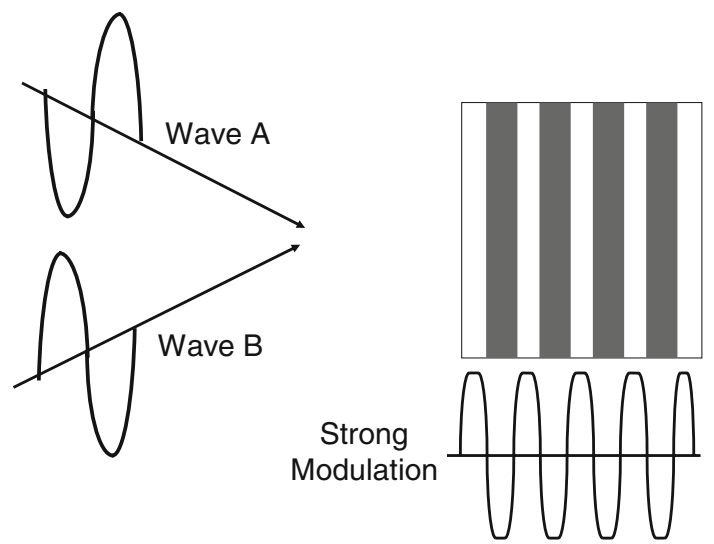

Fig. 7 Signals of equal amplitudes (wave A and wave B) generate an interference pattern with strong or deep modulation

simply selecting one specific input, which will basically retrieve only the according (associatively linked) output. Holographic storage dissipates information across wide areas of the storage medium. Consequently identical areas to store different information are utilized.

The strength of a retrieved signal depends on the modulation depth of the stored interference pattern. Weak or no modulation does prevent a signal from being retrieved by an input signal. As shown in Fig. 7, both signals (input and output) to be stored associatively require similar signal strength (amplitude) in order to form an interference pattern with strong modulation. If the signals are of different strength, modulation is weak as shown in Fig. 8, and no associative linkage will take place.

Hyper-stimulation by shock waves may be considered as storing the specific strong sensor input (pain) with the weak motor output interference pattern once again with weak modulation. This weak (new) pattern overwrites the (old)
Waves of Different Amplitudes
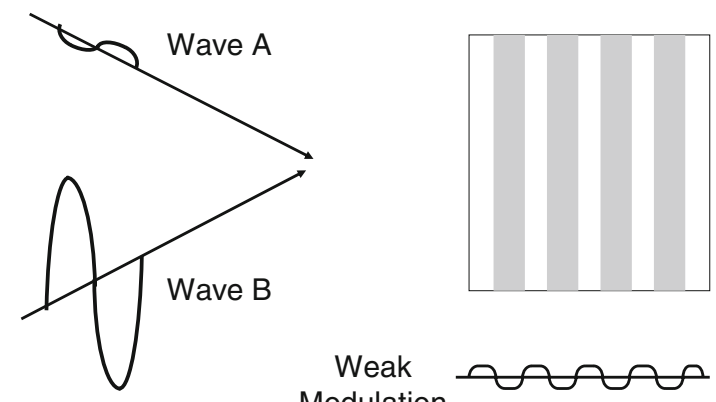

Weak Modulation

Fig. 8 Small signals (wave A) interfere with strong signals (wave B) and generate weak interference patterns with low modulation depth

strong pattern and is stored instead of the old one. Permanent storage is facilitated by stimulating over a time period of some 15-20 min and by periodically repeated treatment sessions as frequently done in ESWT.

Shock wave treatment is a painful procedure. Seen technically, the dynamic range of the neural memory may be exceeded. Figure 9 shows this effect schematically. If the signal strength reaches the saturated part of the response curve, the systems answer (output signal) is small. In other words: the former circulus vitiosus is broken since pain sensation does not any more retrieve muscle/vessel tonus, at least not as strong as before.

The final outcome of the procedure is characterized by a selective "erasure" of the pathological response or reflex pattern. New and normal reflex patterns may develop instead of the previously stored pair of pain and muscle/ vessel tonus. Obviously, associative pain memory considered as the real cause of chronic pain can be selectively reorganized by appropriate shock wave treatment.
Fig. 9 The dynamic range of any storage medium is limited and excessive utilization which may be performed by hyperstimulation results in deformation and degradation of the retrieved signal

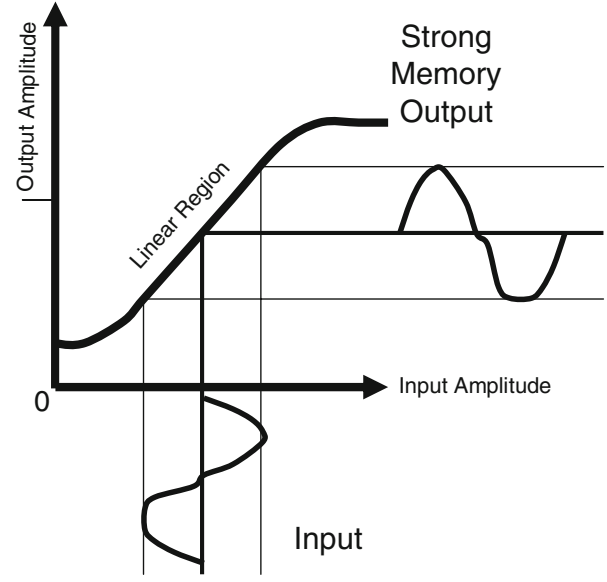

Strong Modulation

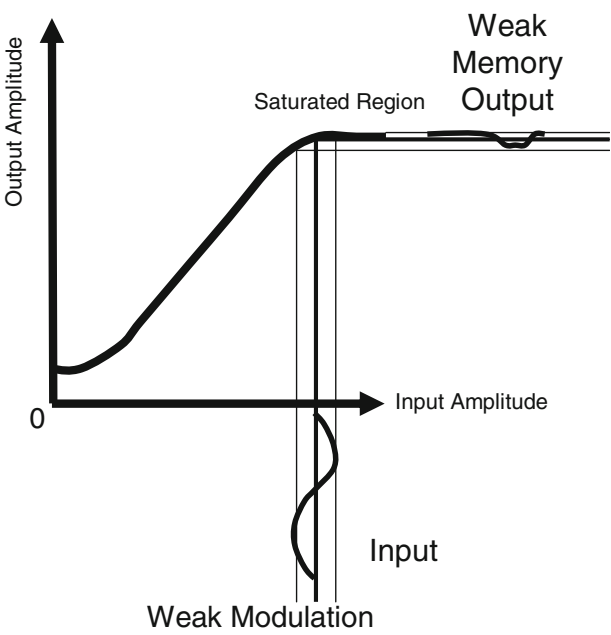


The effect of selective erasing (forgetting) of memory contents matches well the findings of Sandkuehler [25]. According to Sandkuehler slightly painful counter-stimulation is best explained by a long-term depression (LTD) of synaptic strength in nerve fibers.

\section{Discussion}

Associative pain memory linking a specific chronic pain input to particular muscle or vessel tone output may be looked at under two different aspects: first, as a general theory of chronic pain, offering a new insight of pain mechanism and possible new therapeutic regimes and second, as a specific explanation of the therapeutic effect of ESWT and similar treatment modalities.

In the case of ESWT, until now no direct relation of any physical shock wave parameter could be related to the therapeutic effect of ESWT. A consensus group [26] selected a list of physical parameters of a shock wave field such as peak pressure, different focal volumes and related energy contents, energy flux density values for positive and negative pressure contents etc. in order to correlate clinical results with one or the other parameter. Since therapeutic effects could be seen with low energies as well as with high energies, a direct shock wave effect is unlikely. All of the conventional shock wave mechanisms mentioned above would require a more or less linear relation of shock wave dose and treatment effects. On the other hand, two or three repeated treatments at an interval of several days or weeks are often performed in order to enhance the shock wave effects. This behavior leads to the idea of looking for indirect effects such as memory and learning. Obviously, memory effects of the nervous system are not only influenced by the strength of the stimulus but also on predisposition, sensitivity, concentration and attention. Metabolism on the level of synaptic junctions may also be involved.

The theory of associative memory draws the attention from direct interaction of the affected organ to its related memory traces on higher levels of nervous interactions.

In view of this theory, the therapeutic results of ESWT in different heterogeneous indications such as pain diseases, non-unions, induratio penis plastica [27], and ESWT treatment of muscular malfunctions [6] may be considered as reorganization of pathologic reflex patterns. Muscle and/or vessel tone are considered to be affected by intense stimulation of ESWT. Breaking up the pathological reflex bow does not only erase the pathological memory but gives rise to increased circulation and metabolism due to relaxation of vessel tonus. This in turn is taken as a basis for real and permanent healing.

It seems tempting to consider ESWT as a therapeutic tool for the small, but nevertheless clinically extremely demanding group of patients with chronic pain conditions in the urological field. We are, for instance, well aware of the considerable problems that are associated with persistent pain after one or several stone episodes in patients in whom no residuals or no obvious explanation for the pain can be found.

The latter patients might possibly be one group for whom ESWT can be effective.

\section{Conclusion}

The concept of associative pain memory provides a mechanism for chronic pain diseases and explains how ESWT may act on a variety of heterogeneous indications such as close to bone soft tissue pain CPPS, pseudarthrosis, muscular malfunctions and others.

The hypothesis behind is a "malfunction" of the nervous control system due to "pathologic reflexes" which may be conditioned by single overuse or long-term misuse of particular organs or functions. Pathological adaptation to unnatural muscular and vascular tone conditions is assumed to take place within the nervous system on the synaptic level by modification of the synaptic strength of large ensembles of neurons. Adaptation of synaptic threshold patterns is deemed to be the basis of memory functions in general. ESWT with strong and repeated stimulation of synaptic junctions may delete the pathologic memory reflex pattern selectively with respect to the treated pain area. The hypothesis of selective deletion of dedicated pathological reflexes is the charming concept of ESWT. If so, future research needs to show how ESWT interacts with brain functions. It is well known that application of a painful stimulus activates distinctive areas within the brain as proven by modern imaging modalities such as functional magnetic resonance imaging (fMRI) or positron emission tomography (PET). A similar effect should be generated by ESWT and proven accordingly.

Another aspect of future research may be focused on the effect of specific drugs affecting the metabolism of synaptic activity. The healing effect of ESWT should be differently influenced by drugs either facilitating or depressing synaptic thresholds during shock wave application.

The author believes that if the hypothesis presented above can be supported by further research, a new view of chronic pain and related mechanisms may shine a clearing light on several diseases of presently unknown etiology. It may be worth checking the theory on diseases such as Alzheimer, Parkinson, multiple sclerosis (MS) and a wide spectrum of rheumatic diseases.

Open Access This article is distributed under the terms of the Creative Commons Attribution Noncommercial License which permits any 
noncommercial use, distribution, and reproduction in any medium, provided the original author(s) and source are credited.

\section{References}

1. Labek G, Auersperg V, Ziernhöld M, Poulios N, Böhler N (2005) Influence of local anesthesia and energy level on the clinical outcome of extracorporeal shock wave-treatment of chronic plantar fasciitis. Z Orthop Ihre Grenzgeb 143(2):240-246

2. Haist J, von Keitz-Steeger D (1995) Stosswellentherapie knochennaher Weichteilschmerzen, Ein neues Behandlungskonzept. Die Stosswelle, Forschung und Klinik, Attempto-Verlag, 162-165. ISBN 3-89308-228-X

3. Rompe J-D, Küllmer K, Vogel J, Eckardt A, Wahlmann U, Eysel P, Hopf C, Kirkpatrick CJ, Bürger R, Nafe B (1997) Extrakorporale Stosswellentherapie- Experimentelle Grundlagen, klinischer Einsatz. Orthopäde 26:215-228

4. Melzack R, Wall PD (1965) Pain mechanisms: a new theory. Science 150:971-979

5. Wang CJ, Wang FS, Yang KD, Weng LH, Hsu CC, Huang CS, Yang LC (2003) Shock Wave Therapy Induces Neovascularization at the Tendon-Bone Junction. A study in rabbits. J Orthop Res

6. Nishida T, Shimokawa H, Oi K, Tatewaki H, Uwatoku T, Abe K, Matsumoto Y, Kajihara N, Eto M, Matsuda T, Yasui H, Takeshita A, Sunagaea K (2004) Extracorporeal shock wave therapy markedly ameliorates ischemia-induced myocardial dysfunction in pigs in vivo. Circulation, November 9, pp 3055-3061

7. Lohse-Busch H, Kraemer M, Reime U (1997) The use of extracorporeal shock wave fronts for treatment of muscle dysfunction on various etiologies: an overview of first results. In: Siebert W, Buch $\mathrm{M}$ (eds) Extracorporeal shock waves in orthopaedics. Springer, New York, pp 215-230. ISBN 3-540-63092-9

8. Manganotti P, Amelio E (2005) Long-term effect of shock wave therapy on upper limb hypertonia in patients affected by stroke. Stroke 36(9): 1967-1971

9. Russo S, Gigliotti S, de Durante C, Canero R, Andretta D, Corrado B (1997) Results with extracorporeal shock wave therapy in bone and soft tissue pathologies. In: Siebert W, Buch M (eds) Extracorporeal shock waves in orthopaedics. Springer, New York, pp 149155. ISBN 3-540-63092-9

10. Zimmermann R, Cumpanas A, Hoetl L, Janetschek G, Stenzl A, Miclea F (2008) Extracorporeal shock wave therapy for treating chronic pelvic pain syndrome: a fesibility study and first clinical results. BJU Int

11. Schaden W, Thiele R, Kölpl C, Pusch A (2006) Extracorporeal shock waves therapy in skin lesions. ISMST Newsletter, 2(1), pp 13-14, April 2006 (http://www.ISMST.com)

12. Kohonen (1977) Associative memory: a system-theoretical approach. Springer, New York. ISBN 0-387-08017-1

13. Willshaw DJ, Buneman OP, Longuet-Higgins HC (1969) Non-holographic associative memory. Nature 222:960-962

14. Reichardt W (1957) Autokorrelations-Auswertung als Funktionsprinzip des Zentralnervensystems. Z Naturforschung 12b:448

15. Lashley KS (1931) Mass action in cerebral function. Science $73: 245$

16. Gabor D (1968) Holographic model of temporal recall. Nature 217:548

17. Gabor D (1969) Associative holographic memories. IBM J Res Dev 13:2-5

18. Greguss P (1968) Bioholography-a new model of information processing. Nature 219:482

19. Longuet-Higgins HC (1968) Holographic model of temporal recall. Nature 217:104

20. Westlake PR (1970) The possibilities of neural holographic processes within the brain. Kybernetik 7:129-153

21. Mager HJ, Wess O, Waidelich W (1973) Sequential associative information storage and reconstruction in a holographic circuit. Optics Commun 9(2):156-160

22. Wess O, Röder U (1977) A holographic model for associative memory chains. Biol Cybern 27:89-98

23. Cray CM, König P, Engel AK, Singer W (1989) Oscillatory responses in cat visual cortex exhibit intercolumnar synchronization which reflects global stimulus properties. Nature 338(6231):334-337

24. Engel AK, Singer W (2001) Temporal binding and neural correlates of sensory awareness. Trends Cogn Sci 5:16-25

25. Sandkuehler J (2000) Learning and memory in pain pathways. Pain 88:113-118

26. Wess O, Ueberle F, Dührssen R-N, Hilcken D, Krauss W, Reuner Th, Schultheiss R, Staudenraus I, Rattner M, Granz B (1997) Working group technical developments, consensus report. In: Chaussy C, Eisenberger F, Jocham D, Wilbert D (eds) High energy shock waves in medicine. Thieme Verlag Stuttgart 59-71. ISBN 313-104831-X

27. Fahlenkamp D (2005) Extracorporeal shock wave therapy (ESWT) for Peyronies's disease. In: Sven Lahme (eds) Peyronie's Disease, UNI-MED Science, pp 63-74 